\title{
An Unusual Cause of Dizziness: Persistent Ground Sickness in Duane Syndrome
}

\author{
Marco Poloni* and Mauro Ceroni \\ ${ }^{1}$ Voluntary researcher, IRCCS Mario Negri, Milan, Italy \\ ${ }^{2}$ IRCCS Mondino, University of Pavia, Pavia Italy
}

Milan, Italy.

Received Date: May 10, 2021

Published Date: May 26, 2021

Abstract

Ground sickness or mal de debarquement persistence for 4 years following an afternoon ship journey in the Sardinian area of Maddalena sea has been observed in a young man with Duane Syndrome. The relationships of this disturbing condition with this specific congenital abnormality are discussed.

\section{Case Presentation}

A young man, 30 years old, was submitted to a neurologic examination due to the persistence of rocking and swaying sensations with unsteadiness which raised after a boat excursion of one afternoon duration in the sea around Maddalena archipelago in Sardinian area. The persistence of dizziness aroused as ground sickness - mal de debarquement (MdD) in presence a second rare disease such as Duane Syndrome (DS) raises the question of a possible relation between these two diseases. Interestingly these are two coexisting "rare diseases". The case here described showed a pure absence of abduction in OD without a restriction of palpebral fissure on abduction, nor reduced visual acuity at binocular vision; he didn't notice any manifestation of amblyopia in OD and didn't showed a turning compensation of the head. He was fully compensated and never had dizziness or similar disturbances until the boat excursion four years ago. The clinical overall visit as well as the neurological examination revealed entirely normal with the exception of oculomotion alteration, present since the birth and diagnosed as Duane Syndrome (DS); with this exception Oculist and ORL examinations were normal; blood controls, imaging and electrophysiologic examination were at the same without any abnormal findings. In conclusion, the Oculist examination diagnosed a Duane Syndrome; The MR imaging of the brain was entirely normal. After the boat trip of half day duration, he begun to feel instability and body oscillations and overall sensations similar to those he felt when he was on board; this vertigo and dizziness, were present for 4 years and became really uncomfortable, with anxiety, depression, joined to job and daily life difficulties.

\section{Background}

- Duane Syndrome (DS) or Duane Retraction Syndrome: Also known as (Stilling-Turk-Duane Syndrome) is a congenital strabismus occurring in isolated or familial forms, described by Alexander Duane in 1905 [1]: in the complete form it consists of -complete or partial loss of abduction, -retraction of globe on abduction, -narrowing of palpebral fissure on abduction, -partial deficiency of adduction, - oblique involvement with attempts on abduction, - upshoot pr downshoot of globe with abduction (Leash Phenomenon), - widening of palpebral 
aperture with abduction, - deficiency of convergence; it has a prevalence of about $1 / 1000$, slightly more common in females, accounts for up to $4 \%$ of strabismus cases, is the most common form of congenital abnormal innervation, appears isolated in most cases $(70 \%$ )or in $30 \%$ of them associated with other congenital alterations as we will see later; it is mainly unilateral in $90 \%$ and sporadic, while in $10 \%$ is bilateral and associated vertical movement abnormalities with a left eye preference. The most commonly accepted pathophysiology in the neurogenic theory suggests the presence of absent or dysplastic nuclear motor neurons with aberrant innervations of the lateral rectus muscles by the oculomotor nerve. The differential diagnosis for DS include any condition that demonstrates strabismus or limitations of extraocular movements. These includes: Okihiro's syndrome (DS plus radial ray defects), Goldenhar syndrome (DS and malformation of the jaw, cheek and ear, on one side), Wildervanck syndrome (DS plus Hlippel-Feil anomaly and deafness), Moebius Syndrome (DS plus facial paresis), HoltOram Syndrome (DS plus upper limbs abnormalities and heart), Morning Glory Syndrome (DS plus optic disk abnormalities), Abducens Nerve Palsy, Brown Syndrome (limited elevation of the eye by malfunction of the superior oblique muscle), Marcus Gunn Jaw Winking Syndrome (blepharoptosis with synkinetic movements of eyelid elevation during mastication), and Congenital Esotropia (a form of strabismus). There are familial cases: Type 1: Autosomal Dominant (locus 8q13); Type 2: Autosomal Dominant (mutation of CHN1 gene at DURS 2 locus 2q31-q32.1) and Type 3: Autosomal Recessive. Molecular genetic testing of CHN1 is recommended only in familial cases. Management includes the use of spectacles or contact lenses or refractive glasses to improve the compensatory head position; the treatment of amblyopia with standard therapy; the use of Botulinum Toxin decreases the amount of deviation and leash phenomenon (upshoot or downshoot of globe with adduction. Surgery cannot cure Duane Retraction Syndrome, but it can correct for the deviation in the primary position, thereby improving a compensatory head position that can occur in some individuals; It can also improve the Leash phenomenon [2].

- Motion sickness and Mal de Debarquement: Acute motion sickness (MS) arises during passive transportation and resolves spontaneously within 1-3 days at the most after the disappearance of the inducing stimulus. The full picture of acute severe motion sickness evolves with symptoms of lightheadedness, physical discomfort, tiredness, periodic yawning, pallor and light dizziness with apparent surround motion and self-motion. An increase in facial pallor is followed by cold sweats, increased salivation, hypersensitivity to smells, occipital head pain, and feelings of pressure in the upper abdomen. Finally, the central symptoms of nausea, retching, and vomiting develop with motor incoordination, loss of drive and concentration, apathy, and fear of impending doom. MS is not only provoked by vestibular "overstimulation" during strong accelerations of the body, but by unfamiliar (i.e., nonadapted) motion stimuli and particularly by intersensory perceptual incongruencies among the visual, vestibular, and somatosensory systems. The most important concept explaining the pathogenesis of MS is the so-called mismatch theory; the decisive trigger is the incongruence of the signals of motion from various sensory channels or the incongruence between expected and actual sensory stimulation. On a ship we can suffer by seasickness, but we can adapt with time; when we return back walking, we can suffer again of a disturbance similar to sea sickness which take the name of groundsickness or MdD, at least until the vestibulum adapt to the new stationary network conditions: . The MdD is a stance and gait unsteadiness with postural vertigo that develops on land after longer ship travel [3,4]. Moeller and Lempert [5] speculated that it has to do with pseudo hallucinations of the "vestibular memory." Such complaints can occur at short notice in the form of sensorimotor aftereffects even in healthy subjects after a long-persisting motion stimulus (e.g., "seamen's legs"). Patients suffering from this condition exhibit impaired postural stability, kinesiophobia [6], and fatigue [7]. This syndrome lasts for months or years with a substantial economic burden [8] and recalls the development of somatoform vertigo similar to that of phobic postural vertigo. Physical means of prevention of MS is adaptation (habituation) by intermittent exposure to the stimulus. This adaptation, however, is only temporary and specific for each type of acceleration, i.e., resistance to seasickness does not protect from flight sickness. If "vestibular training" does not make the subject resistant, his/her head should be kept still during the stimulus, and additional accelerations that are complexly coupled with the vehicle motion should be avoided. Susceptibility is less when lying than when sitting. MS develops above all in closed vehicles or while reading on the back seat of a car, when the body is being accelerated, but a stationary environment is being viewed, which contradicts the labyrinthine stimuli. By maintaining adequate visual control of the vehicle movement, one can significantly reduce MS from that experienced under eyesclosed conditions. Conversely, susceptibility is significantly increased if primarily stationary contrasts fill the field of vision. Anti-vertiginous drugs such as dimenhydrinate (Dramamine) or scopolamine (Transderm Scop) can inhibit the spontaneous activity of the neurons of the vestibular nuclei, thus reducing the susceptibility to MS and to MdD.

- Vertigo: vertigo is a very unpleasant symptom for the patient. Vertigo may be defined as a distorted perception of the position and/or movement of head in the space, accompanied by nausea, vomiting and other vegetative symptoms at times; other supplementary signs may be nystagmus and postural 
deviations with falls. If we consider the structures which give a contribution to our perception of the position and shift of our head in the surrounding and that maintain our balance in the space, we must look to: - vestibular receptors, located in the inner ear and providing the brain with afferents about head position and movement, - visual system which has a dominant position through sight, - proprioceptors, in the spine, legs and feet which send ascending information on position and movement of body segments; we have to remember the contribution of the cerebellar afferents carrying unconscious information and, in a subsidiary way, the exteroceptive sensitive system too: remember the contribution to the stance of walking in the sand and sea!. When sight, vestibular, proprioceptive, cerebellar and exteroceptive afferents give a concordant information to the nervous system everything sounds well with feeling of balance; when the information along the channels is discordant, something wrong happens, and we feel unhappy and have a sensation of unbalance and dizziness [3].

\section{Discussion}

As concerns the MdD in a patient affected by DS, it seems to much easy to explain the appearance of vertigo through psychogenic solution and I prefer to seek for an abnormality of physiologic mechanisms. Dizziness is present in various conditions of visionrelated problems, including eyestrain, incorrect prescription of eyeglasses or lens, binocular vision disturbances, double vision and nystagmus. It is well known that several conditions favour physiologic dizziness and unbalance: 1) If we are looking to a large object engaging to a wide extent our visual field when it moves, we feel uncertain if what is moving is the object or ourselves, and we feel unstable; we are steady, but the sight give a movement information; 2) When we are carried by a car or by train or plane we are moving, and our vestibular system is stimulated, but our sight and our proprioception give information of absence of movement, above all if we are reading or concentrated on a particular job; we begin to suffer motion sickness (MS) and feel nausea and vomiting; 3) If you go to skiing in a bad weather day with fog, cloudy sky and snow, you really don't have any reference and everything around you is white and you leave completely the orientation in the space and feels very badly with nausea and vomiting; in these situations, is necessary to concentrate on proprioception and seek the aid of exteroceptive stimuli to gain information. Vestibular receptor is very sensible but fortunately has the characteristics of being plastic and adaptable too: the correct name of this propriety is habituation. We have illustrated just few examples of what we can call physiological vertigo, or pseudo-vertigo, simply due to a variant information through the different afferents responsible to integrate our steadiness in the space in normal situations. There are, also, examples of habituation and training: certain boys have fun revolving on themselves until they fall; the Darvish dancers revolve giddy for 10-20 min or more during their exhibitions; who practices the circular bound flight is used to flight in this way an aero model. Now, if you put attention to the different and very intricate modalities through which the pertinent information reaches our brain you can even find the key to solve the problem of your patient: something went wrong in vestibular apparatus and the pattern of stimuli became discordant between the two sides? Or, due to the numerous connections and intricate pathways of vision, vestibular, cerebellar, proprioceptive and exteroceptive afferents you can consider the variety of dysfunctions and diseases which affects this extremely complex network: I wish just recall a couple of strange and unexpected causes of vertigo which I had the chance to observe: one was a colleague who felt dizziness in the morning at the beginning of own work; no information was gained by careful history and objective examination, until he remembered that had changed the lenses of the spectacles! Another patient felt dizzy when he exceeded the speed of $100 \mathrm{Km} / \mathrm{h}$ on the highway towards Milan; here, I was uncertain if the problem was sight related due to the rapid change of images or if it was psychogenic and fear connected, a sort of already cited kinesiophobia; another particular case was a retired man who had a mastoid fistula rear to the right ear; he felt vertigo when he had a dive or a dip in the sea: here the problem was most probably linked to a vestibular stimulus induced by a sudden drop of temperature near the semicircular channels with consequent convective motion of the endolymphatic fluid; It was like a real life Barany Test! Last case was that of two brothers, a female and a man, who were always disturbed by sensations of instability and unsteadiness; both were found affected by familial hemochromatosis and that was the origin of derangement of labyrinthic function; the successful treatment was based in these cases on periodic blood-extraction. DS should lead to dizziness and vertigo, due to the visual disturbances of double vision, and we have seen how much important appears the sight in the symptom of vertigo; moreover, anisometropia and amblyopia were highly prevalent in DS, with the latter generating the former in a significant association [7]. The duration of MdD has been described lasting from 6 months to 10 years (mean 3,5 years) in a survey [10]. Cases of motion triggered (MT) DS and non MT DS have been described [11] with a duration variable, with a mean of 35,4 months (mean 12; range 1-204) for MT and. 82,8 (mean 62,9; range 18-255) for non MT. Interestingly, metabolic and functional connectivity changes have been described [12] in MdD syndrome characterized by an association between entorhinal cortex and amygdala providing both a clearly objective brain modification in an entirely subjective discomfort and both a natural model to study neuroplasticity of the motion adaptation system in humans. The troubling symptoms may find a compensatory action by counterbalanced head turn and by the gradual onset of amblyopia; in this case nor diplopia neither amblyopia were present, most probably due to a compensation. In occasion of the onset of vertigo and dizziness due to MdD the symptoms of dizziness might be unmasked; in other words the equilibrium achieved since the presence of this problem from birth was breached off by the boat trip and the appearance of MdD with its peculiar sensorial manifestations; the introduction 
of a further mismatch between the different factors responsible of balance on a saturated ground lead to the impossibility to find a further compensatory solution. Obviously, it is well known that panic attacks of phobic-obsessive neuroses may generate vertigo, a condition called Kinesiphobia, and that psychosomatic disturbances in patients affected by different forms of anxiety, depression and unhappiness may give a significant contribution to the onset of vertigo and dizziness [13]; nevertheless, a similar contribution without the comprehensive characteristics of mood and personality in the patient here described seems quite unlikely. A dynamic of this type or the coexistence of different factors in the origin of this peculiar disturbance can't obviously be ruled out.

\section{Conclusion}

The Oculist examination diagnosed a Duane Syndrome; The MR imaging of the brain was entirely normal. After the boat trip of half day duration, he begun to feel instability and body oscillations and overall sensations similar to those he felt when he was on board; this vertigo and dizziness, were present for 4 years and became really uncomfortable, with anxiety, depression, joined to job and daily life difficulties.

\section{Acknowledgement}

None.

\section{Conflict of Interest}

No conflict of interest.

\section{References}

1. Alexander Duane (1996) Congenital deficiency of abduction, associated with impairment of adduction, retraction movements, contraction of the palpebral fissure and oblique movements of the eye. 1905 Arch Ophthalmol 114: 1255-56

2. T Brandt, M Dieterich, M Strupp (2013) Vertigo and Dizziness - Common Complaintsm Springer-Verlag, London.

3. Y-H Cha (2009) Mal de Debarquement: Semin Neurol 29(5): 520-527.

4. L. Moeller, Lempert T (2007) Mal de debarquement: pseudohallucinations from vestibular memory? J Neurol 254(6): 813-815.

5. Clark BC, Leporte A, Clark S, Hoffman RL, Quick A, et al. (2013) Effects of persistent Mal de debarquement syndrome on balance, psychological traits, and motor cortex excitability. J Clin Neurosci 20(3): 446-450.

6. Hossaen Hassan Ahmad Ali Alami (2019) Prevalence of anisometropia and amblyopia in Duane Retrtaction Syndrome of patients attended ibn Al-Haitham teaching Eye Hospital, Rev Latnoamericana de Hipertension 14(3): 260-264.

7. BC Clark, A LePorte, SC Richard, L Hoffman, A Quick, et al. (2013) Thomas, Effects of persistent Mal de debarquement syndrome on balance, psychological traits, and motor cortex excitability. J Clin Neurosc 20(3): 446-450.

8. Macke A, LePorte A, Clark BC (2012) Social, societal, and economic burden of mal de debarquement syndrome. Journal of Neurology 259 : 1326-1330.

9. TH Hain, PA Anna, MA Rheinberger (1999) Mal de Debarquement. Arch Otolaryngol Head Neck Surg. 125: 615-620.

10. YH Cha, YY Cui, RW Baloh (2018) Comprehensive clinical profile of Mal de Debarquement Syndrome, Frontiers in Neurology 9: 261.

11. YH Cha, S Chakrapani, A Craig, RW Baloh (2012) Metabolic and Functional Connectivity Changes in Mal de Debarquement Syndrome. PLOSone 7(11): e49560

12. MA Arroll, EA Attree, YH Cha, CP Dancey (2014) The relationship between symptom severity, stigma, illness intrusiveness and depression in Mal de Debarquement Syndrome Journal of Health Psychology 21: $1-12$. 